\title{
THE LOCALIZATION OF THE STRICT TOPOLOGY VIA BOUNDED SETS ${ }^{1}$
}

\section{J. R. DORROH}

The $\beta$ or strict topology on the space $C(S)$ of bounded continuous functions on a locally compact Hausdorff space $S$ was introduced by Buck, see [1]. The purpose of this note is to prove:

THEOREM. No properly stronger locally convex topology on $C(S)$ agrees with $\beta$ on norm bounded sets (equivalently, on $\beta$-bounded sets).

The most obvious consequence of this is that a linear transformation from $C(S)_{\beta}$ into a locally convex space is continuous if it is continuous on bounded sets, a fact which was observed for linear functionals by Buck in [1]. The $\beta^{\prime}$ or bounded strict topology is the strongest locally convex topology on $C(S)$ which agrees with $\beta$ on bounded sets. For a proof of existence, see [2], [5], or [7], where an explicit neighborhood base is given. Our theorem may now be stated: $\beta^{\prime}=\beta$. Thus, since $\beta$ agrees with the compact open topology on norm bounded sets, we have that $\beta$ is the strongest locally convex topology on $C(S)$ which agrees with the compact open topology on norm bounded sets. It is easy to show (see [5]), that $C(S)_{\beta}$, has the same adjoint as $C(S)_{\beta}$, namely the functionals given by elements of $M(S)$, the space of bounded regular Borel measures on $S$. This immediately gives $\beta^{\prime}=\beta$ if $S$ is paracompact, as Conway showed in [4] that $C(S)_{\beta}$ is a Mackey space then. In [3], the $\beta^{\prime}$ topology is discussed in the context of general localizations (see [6, pp. 154 and 155] or [2, part 2]). In [5] and [7], the $\beta^{\prime}$ topology was useful in questions of continuity and equicontinuity of operators, and in neither paper was it apparent that $\beta$ would have served as well.

Proof of Theorem. Let $\|$ denote the supremum norm on $C(S)$ and the variation norm on $M(S)$. Let $C_{0}(S)$ denote the space of functions in $C(S)$ which vanish at infinity, and for $\phi \in C_{0}(S)$, let

$$
V_{\phi}=\{f \in C(S):\|f \phi\| \leqq 1\} .
$$

Then the sets $\left\{V_{\phi}\right\}$ form a neighborhood base at 0 for $\beta$. If $\psi \in C(S)$ and $\mu \in M(S)$, then $|\mu|$ denotes the variation (measure) of $\mu$, and $\psi \mu \in M(S)$ is defined by $\left[\psi_{\mu}\right](E)=\int_{E} \psi d \mu$.

Presented to the Society, on November 18, 1967; received by the editors October 23, 1967.

1 This work was partially supported by NASA grant number 19-001-024. 
If $\psi \in C(s), \psi \geqq 0$, then let

$$
A_{\psi}=\{f \in C(S):|f| \leqq \psi\} .
$$

We see that the polar of $A_{\psi}$ is given by

$$
\left(A_{\psi}\right)^{0}=\{\mu \in M(S):\|\psi \mu\| \leqq 1\} .
$$

Now let $W$ be an absolutely convex $\beta^{\prime}$-closed $\beta^{\prime}$ neighborhood of 0 . For each $n=1,2, \cdots$, let $B_{n}$ denote the closed norm ball in $C(S)$ of radius $n$, and let $\phi_{n} \in C_{0}(S)$ be such that $\phi_{n} \geqq 0$ and

$$
W \cap B_{n} \supset B_{n} \cap V_{\phi_{n}} \text {. }
$$

Let $\phi_{n}{ }^{\prime}=\max \left\{\phi_{n}, 1 / n\right\}, \psi_{n}=1 / \phi_{n}{ }^{\prime}$, and $A_{n}=A_{\psi_{n}}$. Then

$$
W \cap B_{n} \supset A_{n}=B_{n} \cap V_{\phi_{n}} \text {. }
$$

Let $W^{\prime}$ denote the $\beta^{\prime}$-closed absolutely convex hull of $U A_{n}$. Then $W^{\prime} \subset W$, and $\left(W^{\prime}\right)^{0}=\bigcap\left(A_{n}\right)^{0}$. We will show that $\left(W^{\prime}\right)^{0}$ is $\beta$-equicontinuous, so that $W^{\prime}$ is a $\beta$ neighborhood of 0 .

Since each $\left(A_{n}\right)^{0}$ is norm bounded, $\left(W^{\prime}\right)^{0}$ is norm bounded. Suppose $\epsilon>0$. Let $(1 / n)<\epsilon$, and

$$
K=\left\{x \in S:\left|\phi_{n}(x)\right| \geqq 1 / n\right\} .
$$

If $\mu \in\left(W^{\prime}\right)^{0}$, then $\mu \in\left(A_{n}\right)^{0}$, so that

$$
\begin{aligned}
|\mu|(S \backslash K) & =\int_{S \backslash K} d|\mu|=\int_{S \backslash K} \phi_{n}^{\prime} \psi_{n} d|\mu|=\int_{S \backslash K} \phi_{n}^{\prime} d\left|\psi_{n} \mu\right| \\
& =(1 / n) \int_{S \backslash K} d\left|\psi_{n} \mu\right| \leqq(1 / n)\left\|\psi_{n} \mu\right\|<\epsilon .
\end{aligned}
$$

Thus, $\left(W^{\prime}\right)^{0}$ is $\beta$-equicontinuous by Conway's characterization in [4].

\section{REFERENCES}

1. R. C. Buck, Bounded continuous functions on a locally compact space, Michigan Math. J. 5 (1958), 95-104.

2. H. S. Collins, Completeness and compactness in linear topological spaces, Trans. Amer. Math. Soc. 79 (1955), 256-280.

3. H. S. Collins and J. R. Dorroh, Remarks on certain function spaces, Math. Ann. 176 (1968), 157-168.

4. J. R. Conway, The strict topology and compactness in the space of measures. II, Trans. Amer. Math. Soc. 126 (1967), 474-486.

5. J. R. Dorroh, Semi-groups of maps in a locally compact space, Canad J. Math. 19 (1967), 688-696.

6. H. H. Schaefer, Topological vector spaces, Macmillan, New York, 1966.

7. F. D. Sentilles, Kernel representations of operators and their adjoints, Pacific J. Math. 23 (1967), 153-162.

Louisiana State University 